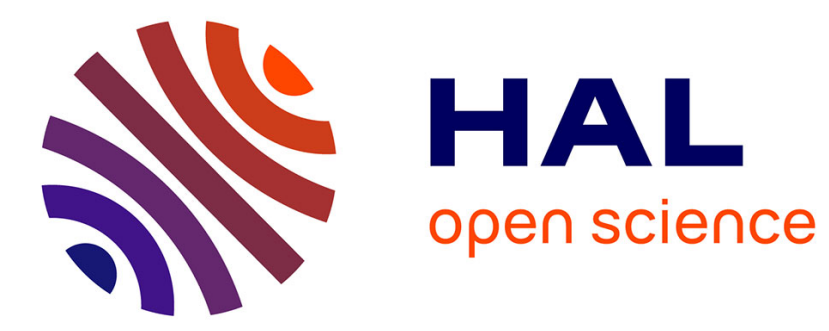

\title{
Generalized inverses applied to Pulse Width Modulation for static conversion: a first study
}

Paul-Etienne Vidal, Simon Cailhol, Frédéric Rotella, Karima Berkoune, Ana-Maria Llor, Maurice Fadel

\section{- To cite this version:}

Paul-Etienne Vidal, Simon Cailhol, Frédéric Rotella, Karima Berkoune, Ana-Maria Llor, et al.. Generalized inverses applied to Pulse Width Modulation for static conversion: a first study. EPE'13 ECCE Europe, Sep 2013, Lille, France. pp.1-10, 10.1109/EPE.2013.6634683 . hal-00952708

\author{
HAL Id: hal-00952708 \\ https://hal.science/hal-00952708
}

Submitted on 27 Feb 2014

HAL is a multi-disciplinary open access archive for the deposit and dissemination of scientific research documents, whether they are published or not. The documents may come from teaching and research institutions in France or abroad, or from public or private research centers.
L'archive ouverte pluridisciplinaire HAL, est destinée au dépôt et à la diffusion de documents scientifiques de niveau recherche, publiés ou non, émanant des établissements d'enseignement et de recherche français ou étrangers, des laboratoires publics ou privés. 


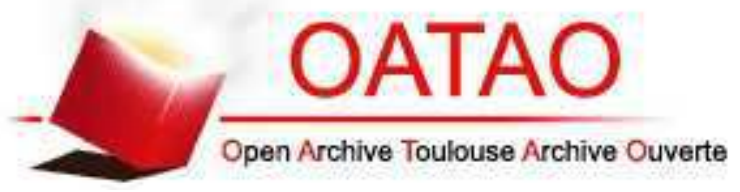

\section{Open Archive Toulouse Archive Ouverte (OATAO)}

OATAO is an open access repository that collects the work of Toulouse researchers and makes it freely available over the web where possible.

This is an author-deposited version published in: http://oatao.univ-toulouse.fr/ Eprints ID: 10730

\section{To cite this version:}

Vidal, Paul-Etienne and Cailhol, Simon and Rotella, Frédéric and Berkoune, Karima and Llor, Ana-Maria and Fadel, Maurice Generalized inverses applied to Pulse Width Modulation for static conversion: a first study. (2013) In: EPE'13 ECCE Europe, 03 September 2013 - 05 September 2013 (Lille, France). 


\title{
Generalized Inverses applied to Pulse Width Modulation for static conversion: a first study
}

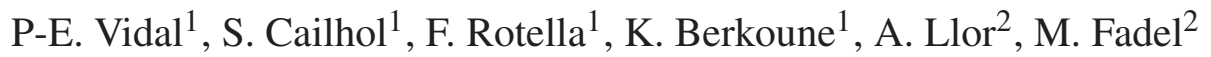 \\ ${ }^{1}$ Universite de Toulouse, Laboratoire Genie de Production (LGP) \\ INPT/ENIT, 47 avenue d'Azereix, BP 1629, 65016 Tarbes Cedex, France \\ ${ }^{2}$ Universite de Toulouse, LAPLACE-CNRS, UMR 5213 \\ INPT/ ENSEEIHT, 2 rue Charles Camichel, BP 7122, 31071 Toulouse, Cedex 7, France \\ Phone: +33 (5) 62442700 \\ Corresponding author : paul-etienne.vidal@enit.fr \\ URL: http://www.enit.fr/fr/recherche.html
}

\section{Keywords}

$<<$ Converter control $>><<$ Device modeling $>><<$ Energy efficiency $>><<$ Modulation Strategy $>>$ $<<$ Pulse Width Modulation $>><<$ Voltage Source Inverter $>>$

\begin{abstract}
This paper points out the generic matrix approach to design Pulse Width Modulation strategies of threephase Voltage Source Inverters. This well-known problem has infinitely many solutions, and many modulation methods already exist. This mathematical approach deserves to be explored by its rigor and must identify known but also new solutions.
\end{abstract}

\section{Introduction}

The static converters are electrical components that can be found in many different applications. In high power devices, such as railway applications, air-planes but also automotives one can find DC to AC converters (named inverters). Such a device allows to connect, in Voltage Source Inverter (VSI) configurations, DC voltage sources to AC current sources. Current sources are mainly constituted of electrical machines or resistive - inductive loads, (Fig. 1).
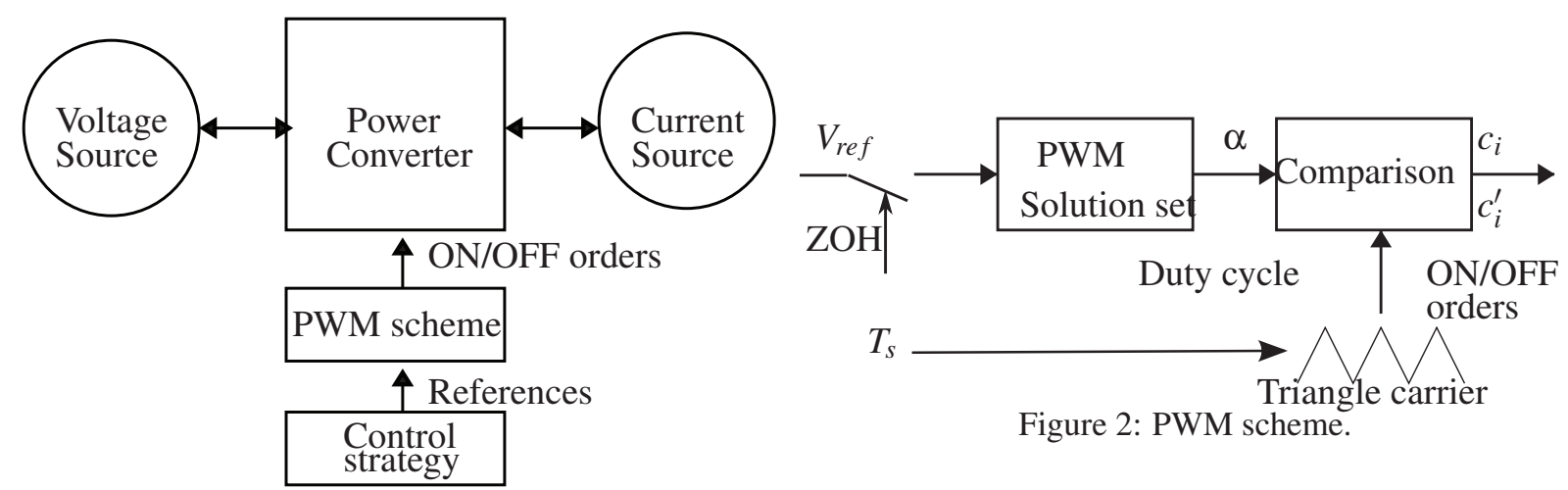

Figure 1: Direct static converter.

Beside the control strategy box, which ensures the closed loop control, a specific stage is distinguished. It is situated between the power converter device and the control board. Indeed this specific stage translates the continuous reference given by the closed loop feedback in binary signals. Finally, they are applied to the power device as illustrated in Fig. 2. In most cases this stage is based on Pulse Width Modulation (PWM) technique. The PWM uses the average value of the voltage applied to the load in order to check the output desired voltage. In most applications, this output voltage will ideally be a sine wave. 
In power electronics, the PWM goal is to modulate the magnitude, the frequency and the phase of voltages or currents. These informations are contained in the reference signal issued from the control stage. In the original PWM technique applied to VSI, a sinusoidal reference voltage is compared to a triangle carrier. The output is ON/OFF order applied to the switches. K. B. Bose made an overview of several PWM techniques following, the current or voltage controller, the feed-forward or feedback methods, carrier or non-carrier based ones, etc. [1]. Some of them are considered as continuous: the modulation waves are always within the triangle peak boundaries. In such a case, for each carrier period, triangle and modulation waves intersect to produce ON/OFF switching orders. Others PWM methods are said discontinuous: for instance one leg is clamped at the $D C$ voltage value for more than one switching period. In some studies, graphical tools are displayed in order to aid the design, the performance evaluation etc. [2]. Other studies detail how to increase the PWM efficiency [3], or what are the link between different PWM methods [4], [5]. Some authors also work on the interaction between the control and the PWM technique in order to improve motor efficiencies [6]. Nowadays the trend is to apply usual PWM to multilevel converters, [7] [8]. It appears to our knowledge that, a generic link between the used VSI model and a formal mathematical approach leading to the PWM solution set has never been described. Let us consider now the carrier based PWM technique. It consists in the comparison of reference waveform $\alpha$, linked to the duty cycle, and one triangle carrier. It is assumed that the reference voltage, $V_{r e f}$, is issued from regulators and that the control strategy is already done. The ON/OFF orders, $c_{i}, c_{i}^{\prime}$, are applied to power switches of the three-phase VSI leg, as illustrated in Fig. 2. The goal of this study is to provide a new and generic mathematical solution of PWM - triangle based carrier. From the VSI model, a specific inversion will lead to the PWM solution set. Finally, the study checks that some of the well known PWM strategies are obtained by fixing some free parameters. The boundaries of the freedom parameters are detailed. Moreover, some simulation results are provided.

\section{Model of $3 \varphi$ Voltage Source Inverter}

\section{VSI average model}

Let us consider a three-phase VSI as described in Fig. 3. $E$, is a $D C$ voltage. Each ideal switch, denoted $K_{i}$ or $K_{i}^{\prime}$, with $i \in\{1,2,3\}$ is considered to be assigned an ON/OFF control signal $c_{i} c_{i}^{\prime} \in\{0,1\}$ respectively. Let us remind that

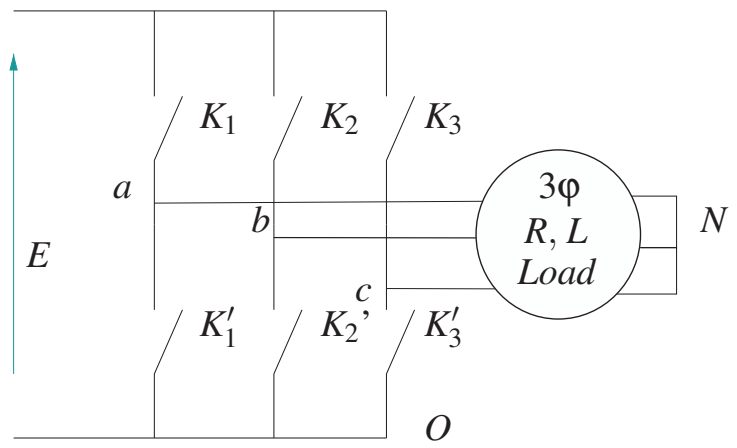

Figure 3: Three-phase voltage source inverter.

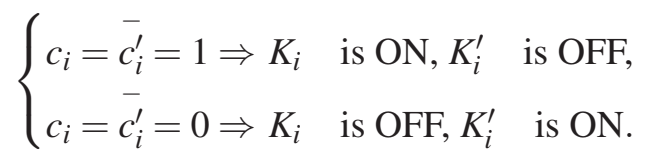

For each ideal switch, the duty cycle applied on a switching period $T_{s}$, is defined as

$$
\alpha_{i}=\frac{t_{i_{O N}}}{T_{s}} \quad \text { with } \quad i \in\{1,2,3\}
$$

where $t_{i_{O N}}$ is the time when $K_{i}$ is switched on. Then the mean value of the line voltages $V_{a O}(t), V_{b O}(t), V_{c O}(t)$ is expressed as

$$
<V_{i O}(t)>_{T_{s}}=\frac{1}{T_{s}} \int_{0}^{T_{s}} V_{i 0}(t) d t=\alpha_{i} E .
$$

For simplicity sake, we assume that the $3 \varphi R-L$ load is balanced, $Z=Z_{1}=Z_{2}=Z_{3}$ with $Z$ the complex impedance, and star-connected. An average model of the inverter is considered and each average voltage 
$<V_{i O}(t)>_{T_{s}}$ is denoted as $V_{i O}=\alpha_{i} E$. The load neutral voltage is given by

$$
V_{N O}(t)=\frac{1}{3}\left(V_{a O}(t)+V_{b O}(t)+V_{c O}(t)\right)=\frac{1}{3}\left[\begin{array}{lll}
1 & 1 & 1
\end{array}\right] V_{l O}(t)
$$

with $V_{l O}(t)=\left[\begin{array}{lll}V_{a O}(t) & V_{b O}(t) & V_{c O}(t)\end{array}\right]^{T}$. Using the average expression $V_{i N}=V_{i O}-V_{N O}$, the load voltages are expressed as

$$
V_{l N}=\left[\begin{array}{lll}
V_{a N} & V_{b N} & V_{c N}
\end{array}\right]^{T}=V_{l O}-\left[\begin{array}{lll}
1 & 1 & 1
\end{array}\right]^{T} V_{N O} .
$$

Taking into account (3), in equations (4) and (5), leads to

$$
V_{l N}=\frac{E}{3}\left[\begin{array}{ccc}
2 & -1 & -1 \\
-1 & 2 & -1 \\
-1 & -1 & 2
\end{array}\right] \alpha .
$$

Eq. (6) relates the load voltage average and the duty cycle $\alpha$, if

$$
\alpha=\left[\begin{array}{lll}
\alpha_{1} & \alpha_{2} & \alpha_{3}
\end{array}\right]^{T} .
$$

Consequently,

$$
V_{l N}=\frac{E}{3} M \alpha \quad \text { with } \quad M=\left[\begin{array}{ccc}
2 & -1 & -1 \\
-1 & 2 & -1 \\
-1 & -1 & 2
\end{array}\right] .
$$

\section{VSI simplified model}

Because of

$$
V_{a N}+V_{b N}+V_{c N}=0
$$

thus,

$$
\operatorname{rank}(M)=\operatorname{rank}\left(\left[M V_{l N}\right]\right)=2 .
$$

Following usual results on linear system [9], the expression (8) is reduced to

$$
W=N \alpha,
$$

where $W=\left[\begin{array}{l}V_{a N} \\ V_{b N}\end{array}\right]$ and $N=\frac{E}{3}\left[\begin{array}{ccc}2 & -1 & -1 \\ -1 & 2 & -1\end{array}\right]$. This expression is a compatible system with an infinite number of solutions. Indeed, the VSI simplified model is finally obtained. It links the load voltages and the duty cycles with the non square matrix $N$.

The regulation strategy provides the load voltage reference vector, $V_{r e f}=\left[V_{r e f_{a N}} V_{r e f_{b N}} V_{r e f_{c N}}\right]^{T}$. So that, $W_{\text {ref }}$ is expressed by

$$
W_{r e f}=\left[\begin{array}{c}
V_{r e f} \\
V_{r e f} \\
r_{b N}
\end{array}\right] .
$$

Due to singularity of the $N$ matrix, we cannot obtain the reference duty cycle by applying the usual inverse. Effectively, it does not exist. The purpose of the next section is to overcome this drawback.

\section{Generalized Inverse theory, applied to VSI}

\section{Short survey of Generalized Inverse theory}

It is well known that each square and non-singular matrix $A$ has a unique inverse, named $A^{-1}$, which satisfies

$$
A A^{-1} A=A \text {. }
$$


At the beginning of the $X X^{\text {th }}$ century, needs for some kind of generalized inverse were pointed out for differential operator [10]. For every matrix, a generalized inverse, $A^{[1]}$, is defined such as

$$
A A^{[1]} A=A .
$$

Some features of the generalized inverse are mentioned [9]: it exists for every matrices; it has some of the properties of the usual inverse; when $A$ is non-singular, $A^{[1]}=A^{-1}$; it is not unique.

To overcome the last point, based on work of Moore [11], [12] and Penrose [13], the Moore-Penrose inverse or pseudo-inverse of every matrix $A$, denoted, $A^{\dagger}$ is defined. $A^{\dagger}$ satisfies the four Penrose equations

$$
\begin{aligned}
A A^{\dagger} A & =A & & A^{\dagger} A A^{\dagger}=A^{\dagger} \\
\left(A A^{\dagger}\right)^{*} & =A^{\dagger} A & & \left(A^{\dagger} A\right)^{*}=A^{\dagger} A
\end{aligned}
$$

where $A^{*}$ denotes the conjugate transpose of $A$. The main property of $A^{\dagger}$ is that for every matrix $A, A^{\dagger}$ is unique. It is obvious that $A^{\dagger}$ is a particular generalized inverse of $A$. The main application of generalized inverse theory is to get the solution set of linear systems [14]. Let us consider a linear system described by

$$
A X=B
$$

where $A$ is a $[n * m]$ matrix and $B$ is a $[n * p]$ matrix. Then, if (17) is compatible, the general solution of (17) is

$$
X=A^{[1]} B+\left(I_{m}-A^{[1]} A\right) Y
$$

where $Y$ is an arbitrary $(m * p)$ matrix and $A^{[1]}$ is a generalized inverse of $A$. It is obvious that $Y$ can be chosen in order to satisfy at the outset, some fixed constraints. The $A^{[1]} B$ part in (18) stands for the basis solution, namely obtained with $Y=0$. It depends on the particular choice for $A^{[1]}$. As $A^{\dagger}$ is a particular generalized inverse, the solution set (18) is generated as

$$
X=A^{\dagger} B+\left(I_{m}-A^{\dagger} A\right) Z
$$

where $Z$ is an arbitrary $(m * p)$ matrix. Despite the fact that the basis solution in (18) and (19) is distinct, the solution sets (18) and (19) are identical. The interest of considering $A^{\dagger}$ is to benefit of stable numeric algorithms to obtain this unique matrix [15]. We must insist here that the dimension of the solution space is given by

$$
\operatorname{dim}(\operatorname{Ker} A)=\operatorname{rank}\left(I-A^{[1]} A\right)=\operatorname{rank}\left(I-A^{+} A\right)
$$

\section{Generalized inverse applied to $3 \varphi$ VSI model}

By using the results of the previous part, the solution set of the duty cycle waveforms is obtained. Thanks to (19), the solution set is expressed as

$$
\alpha=N^{\dagger} W+\left(I_{3}-N^{\dagger} N\right) z
$$

where $W=\left[V_{r e f_{a N}} V_{r e f_{b N}}\right]^{T}$ is the output reference voltages given by regulators as illustrated in Fig. 2 . Firstly a global $z$ such as $z=\left[z_{1} z_{2} z_{3}\right]^{T}$ is considered. Then, as $\operatorname{rank}(N)=2, N^{\dagger}$ is given by [9]:

$$
N^{\dagger}=N^{T}\left(N N^{T}\right)^{-1}=\frac{3}{E}\left[\begin{array}{cc}
\frac{1}{3} & 0 \\
0 & \frac{1}{3} \\
-\frac{1}{3} & -\frac{1}{3}
\end{array}\right] .
$$

Finally, this leads to express the duty cycle vector $\alpha$ as

$$
\alpha=\frac{1}{E}\left[\begin{array}{cc}
1 & 0 \\
0 & 1 \\
-1 & -1
\end{array}\right]\left[\begin{array}{l}
V_{r e f a N} \\
V_{r e f_{b N}}
\end{array}\right]+\frac{1}{3}\left[\begin{array}{lll}
1 & 1 & 1 \\
1 & 1 & 1 \\
1 & 1 & 1
\end{array}\right]\left[\begin{array}{l}
z_{1} \\
z_{2} \\
z_{3}
\end{array}\right]=\frac{1}{E}\left[\begin{array}{l}
V_{r e f} \\
V_{r e f} \\
V_{r e f N}
\end{array}\right]+\left[\begin{array}{l}
1 \\
1 \\
1
\end{array}\right] \lambda
$$


where

$$
\lambda=\frac{z_{1}+z_{2}+z_{3}}{3} .
$$

Finally, with $U=\left[\begin{array}{lll}1 & 1 & 1\end{array}\right]^{T}$,

$$
\alpha=\frac{V_{r e f}}{E}+\lambda U
$$

where $\lambda$ is the scalar degree of freedom leading to the solution set. It has to be defined by using the compatibility expression given in (9). Then $\lambda$ is

$$
\lambda=\frac{\alpha_{1}+\alpha_{2}+\alpha_{3}}{3} .
$$

Moreover, for each $i \in\{1,2,3\}$, the duty cycle is included within $0 \leq \alpha_{i} \leq 1$. Then, Eq. (24) implies

$$
-\frac{\min \left(V_{r e f}\right)}{E} \leq \lambda \leq 1-\frac{\max \left(V_{r e f}\right)}{E} .
$$

Nevertheless, the solution obtained in (24) is not completely satisfying because it is not directly linked with most of classical carrier based PWM in use. In next section a method is developed to recover classical solutions.

\section{Translation of the basis solution}

Starting with (24), two new variables $\mu$ and $\lambda_{b}$ are introduced such as

$$
\lambda=\lambda_{b}+\mu \text {. }
$$

Then the general solution is given by

$$
\alpha=\frac{V_{r e f}}{E}+\lambda_{b} U+\mu U=\alpha_{b}+\mu U
$$

with $\alpha_{b}$ the basis solution defined as

$$
\alpha_{b}=\frac{V_{r e f}}{E}+\lambda_{b} U \text {. }
$$

In the following section is detailed that fixing $\lambda_{b}$ and $\mu$ allows to express some well known PWM solutions. According to the given reference voltages $V_{r e f}$, the values or boundaries of the freedom parameters $\mu$ and $\lambda_{b}$ are expressed.

\section{Simulation results}

\section{Simulation context}

Scilab, which is an open source software for matrix calculus and system simulations, is used [16]. The system described in Fig. 3 is simulated using a $3 \varphi R L$ load. A state space model is introduced in order to obtain the line currents

$$
I(t)=A I(t)+B V_{l N}(t) \quad \Leftrightarrow \quad I(t)=-\frac{R}{L} I(t)+\frac{1}{L} V_{l N}(t)
$$

with $R=15 \Omega$ and $L=1 \mathrm{mH}$. The line potential $+E$ or 0 is applied to the $\{a, b, c\}$ line points such as $V_{l N}(t)=\left[V_{a N} V_{b N} V_{c N}\right]^{T}$. It is deduced from the switching binary order applied to each ideal switch. In order to obtain these switching signals, the regular PWM is implemented, as given in Fig. 2. In such a scheme, a $3 \varphi$ load reference voltage is applied,

$$
V_{r e f}(t)=\left[\begin{array}{lll}
V_{\max } \cos (2 \pi f * t) & V_{\max } \cos \left(2 \pi f * t-\frac{2 \pi}{3}\right) & V_{\max } \cos \left(2 \pi f * t-\frac{4 \pi}{3}\right)
\end{array}\right]^{T} .
$$

Their frequencies are fixed, $f=50 \mathrm{~Hz}$, whereas their peak values can be changed, $V_{\max } \in[0 ; 325]$, with $E=562 \mathrm{~V}$. Then the reference voltage is sampled by a zero-order hold. It is noted as $V_{r e f, k}$. Obviously the PWM solution set is obtained by (28). Finally, the duty cycles to apply are compared to a triangle carrier. In this study the carrier frequency is fixed at $10 \mathrm{kHz}$. Consequently, the duty cycle values are constant during each switching period $T_{s}=0.1 \mathrm{~ms}$. 


\section{Sinusoidal PWM}

To check the accuracy of our model, this basic PWM is simulated. It is obtained with

$$
\lambda_{b}=\frac{1}{2} \quad \text { and } \quad \mu=0
$$

in the main duty cycle expression given in equation (28). Finally the duty cycle is expressed as

$$
\alpha=\frac{V_{r e f, k}}{E}+\frac{1}{2} U \text {. }
$$

The sampled duty cycle $\alpha$ illustrated in Fig. 4, is compared to the triangle carrier. In such example, two $V_{\max }$ values are applied:

$$
V_{\max }= \begin{cases}\frac{E}{3} & \text { if } \quad 0 \leq \text { Time }<0.03 s, \\ \frac{E}{2} & \text { if } \quad 0.03 s \leq \text { Time } \leq 0.06 s,\end{cases}
$$

The obtained $3 \varphi$ currents are given in Fig. 5 .

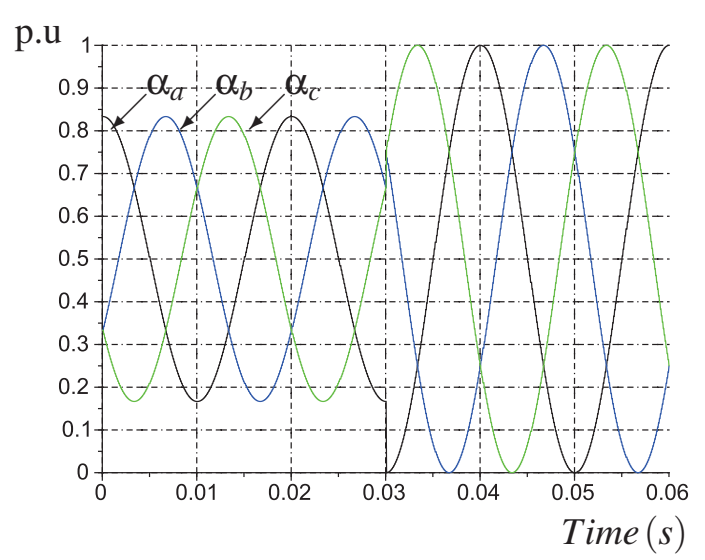

Figure 4: Sampled duty cycle $\alpha$.

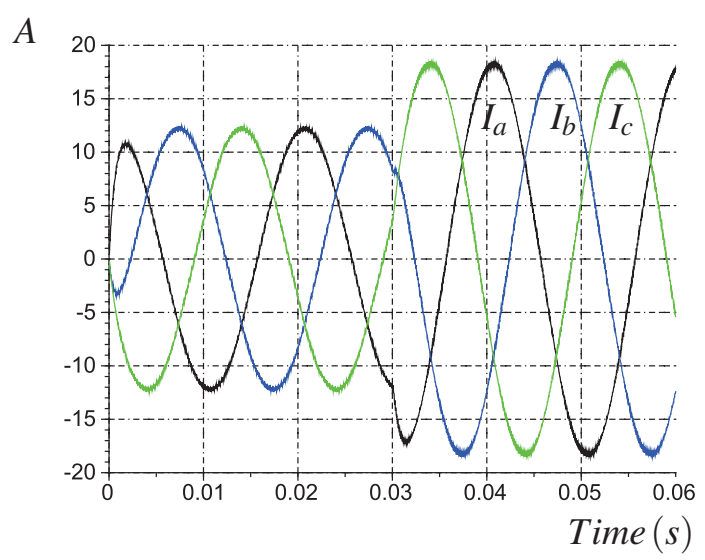

Figure 5: Sinusoidal PWM - $3 \varphi$ currents.

A zoom view of the comparison of the triangle carrier and the sampled duty cycle is provided in Fig. 6 . It is noted that for $V_{r e f, k}$ values higher than $\frac{E}{2}$, the voltage linearity zone is not ensured. The maximal possible value is obtained for $V_{\max }=\frac{E}{2}$. Indeed, each duty cycle achieves his maximum $\alpha_{i}=1$, for appropriate time, as illustrated in Fig. 4.

\section{Zero Sequence Signal PWM}

This technique is used in order to extend the linearity zone of PWM, [17]. Some studies such as [18] reveals that the linearity zone of PWM is linked to the modulation index value. This index allows to quantify the fundamental of the load voltage, and is expressed as

$$
m_{i}=\frac{V_{\max }}{V_{\text {six }- \text { step }}} .
$$

$V_{\text {six-step }}$ is the maximum fundamental value of a square wave.

To obtain the Zero Sequence Signal PWM, a zero sequence voltage $\frac{V_{N O}}{E}$ is added to the duty cycle obtained in a SPWM, Eq. (33). It leads to a new expression of duty cycles

$$
\alpha=\frac{V_{r e f, k}}{E}+\frac{1}{2} U+\frac{V_{N O}}{E} U \text {. }
$$

Considering (28) and (36), it is obvious that

$$
\lambda_{b}=\frac{1}{2} \quad \text { and } \quad \mu=\frac{V_{N O}}{E}
$$




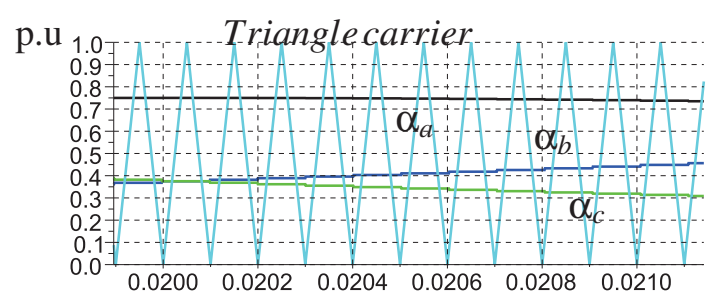

A

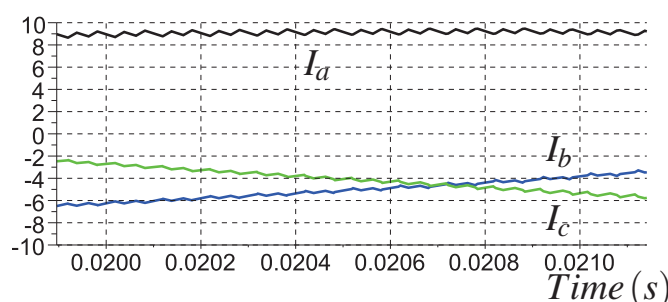

Figure 6: Zoom view - comparison of $\alpha$ triangle carrier, followed by the $3 \varphi$ currents.

fulfill the degrees of freedom of the solution set to obtain (36). The most common case encountered is

$$
V_{N O}=\frac{V_{m e d}}{2},
$$

with $V_{m e d}$ the medium value of the load voltage $V_{l N}$. For instance, if $V_{a N} \leqslant V_{b N} \leqslant V_{c N}$, then $V_{\text {med }}=V_{b N}$. This is expressed as

$$
\frac{V_{m e d}}{2}=-\frac{\max \left(V_{r e f, k}\right)+\min \left(V_{r e f, k}\right)}{2}=V_{N O} .
$$

Then, $V_{\max }$ can reach the maximal line voltage value, $\frac{E}{\sqrt{3}}$. In Fig. 7 the duty cycles applied are illustrated. The $\mu$ term, which corresponds to $\frac{V_{N O}}{E}$ is also displayed. The resulted currents point out the good behaviour of the model.

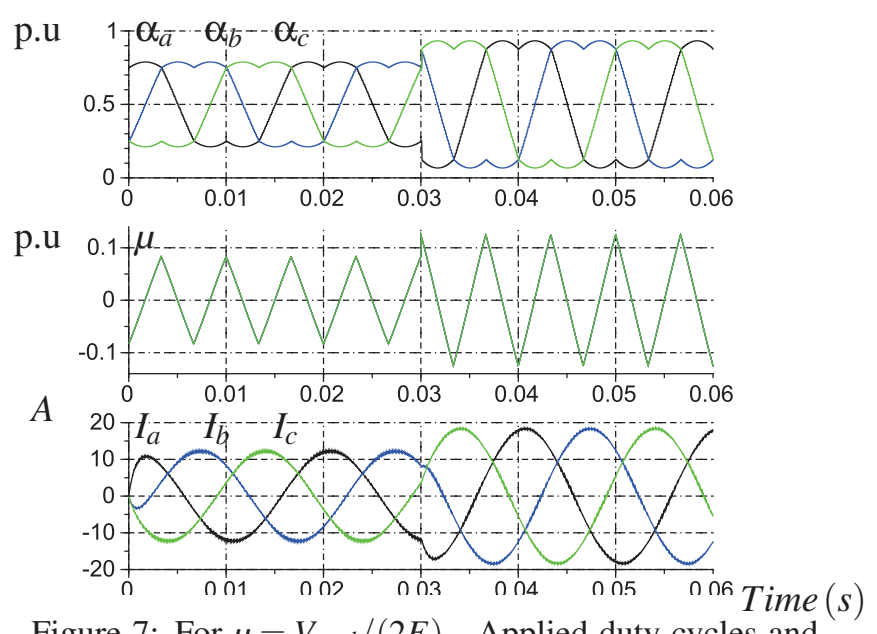

Figure 7: For $\mu=V_{\text {med }} /(2 E)$ - Applied duty cycles and obtained currents.

\section{$\mu$ optimisation}

\section{The linearity voltage zone}

To analyze the $\mu$ optimisation, the linearity voltage zone is defined as the main criterion. It is monitored thanks to the modulation ratio expressed in (35). On the one hand, using a Sinusoidal PWM this ratio is 
known to reach $m_{i}=\frac{\pi}{4} \approx 0.785$. This modulation ratio is reached for $V_{\max }=\frac{E}{2}$. Whereas $V_{\text {six-step }}=\frac{2 E}{\pi}$ is the maximum fundamental value of a square wave. Each output voltage allowed $\in\left\{\frac{-2 E}{3} \frac{-E}{3} \frac{E}{3} \frac{2 E}{3}\right\}$, is maintained during a time period of $\frac{1}{6 f}$. On the other hand, using a Zero Sequence Signal PWM leads to consider a maximal voltage value $V_{\max }=\frac{E}{\sqrt{3}}$. Consequently, the modulation index is increased: $m_{i}=0.907$.

Following this, the sweeping range for $\mu$ is established using (28) and (26). Indeed the degree of freedom is expressed. Considering $\lambda_{b}=\frac{1}{2}$ implies

$$
-\frac{\min \left(V_{r e f, k}\right)}{E}-\frac{1}{2} \leq \mu \leq \frac{1}{2}-\frac{\max \left(V_{r e f, k}\right)}{E} .
$$

Thanks Eq. (40), the high and low margins, $\mu_{\text {high }}, \mu_{\text {low }}$, are expressed

$$
\mu_{\text {low }} \leq \mu \leq \mu_{\text {high }} \text {. }
$$

Each margin is dependent on the maximal or the minimal values of the desired voltage. It is obvious that (40) is true only if

$$
\mu_{\text {low }} \leq \mu_{\text {high }} \quad \Leftrightarrow \quad \max \left(V_{\text {ref }, k}\right)-\min \left(V_{\text {ref }, k}\right) \leq E .
$$

Moreover, $\max \left(V_{r e f, k}\right)-\min \left(V_{r e f, k}\right)$ is the maximal value of the line voltages $V_{a b} V_{b c}$ and $V_{c a}$. It is established by

$$
\max \left(V_{\text {ref }, k}\right)-\min \left(V_{\text {ref }, k}\right)=\max \left(V_{l l}\right)=\sqrt{3} V_{\max },
$$

with $V_{l l}=\left[V_{a b} V_{b c} V_{c a}\right]^{T}$. It is conluded that, for a three-phase VSI as illustrated in Fig. 3, the maximal linearity voltage zone is ensured while the phase voltage involved in the PWM process is

$$
V_{\max } \leq \frac{E}{\sqrt{3}} .
$$

To illustrate such degree of freedom, a first obvious way to ensure (40), is to consider the average point between the two margins such as

$$
\mu=\frac{\mu_{\text {high }}+\mu_{\text {low }}}{2} \text {. }
$$

This leads to express

$$
\mu=-\frac{1}{2 E}\left(\max \left(V_{r e f, k}\right)+\min \left(V_{r e f, k}\right)\right)
$$

which is exactly the $\frac{V_{N O}}{E}$ expression given in Eq. (38) and Eq. (39).

Let us now consider the Sinusoidal PWM (cf. section) and the Zero Sequence Signal PWM (cf. section) simulations. Following (31), the $\mu$ values, $\mu_{S}$ and $\mu_{Z S S}$ respectively, are computed. They are related to the 3 reference peak voltages: $V_{\max } \in\left\{\frac{E}{3} \frac{E}{2} \frac{E}{\sqrt{3}}\right\}$. The result is presented in Fig. 8. This figure is obtained thanks to

$$
V_{\max }=\left\{\begin{array}{lll}
\frac{E}{3} & \text { if } \quad 0 \leq \text { Time }<0.03 s \\
\frac{E}{2} & \text { if } \quad 0.03 s \leq \text { Time }<0.06 s, \\
\frac{E}{\sqrt{3}} & \text { if } \quad 0.06 s \leq \text { Time } \leq 0.1 s .
\end{array}\right.
$$

The last case corresponds to the maximal value allowed in order to reach the maximal value of the modulation index. As illustrated in Fig. 8, it becomes obvious that the Sinusoidal PWM do not match anymore the linearity assessment (40) when $V_{\max }>\frac{E}{2}$. As a consequence, the duty cycles for each PWM reach their maximal value when $V_{\max }=\frac{E}{2}, V_{\max }=\frac{E}{\sqrt{3}}$ respectively, as shown in Fig. 9.

Fig. 8 also highlights that within the $\mu_{\text {high }} \mu_{\text {low }}$ margins, the maximal linearity ratio $m_{i}$ is ensured. It is concluded that all the PWM carrier based solutions are included within the solution set (28). Moreover it is observed that the $\mu$ waveform is not unique. This means that other possible solutions which fulfill other criterions, can be highlight. 

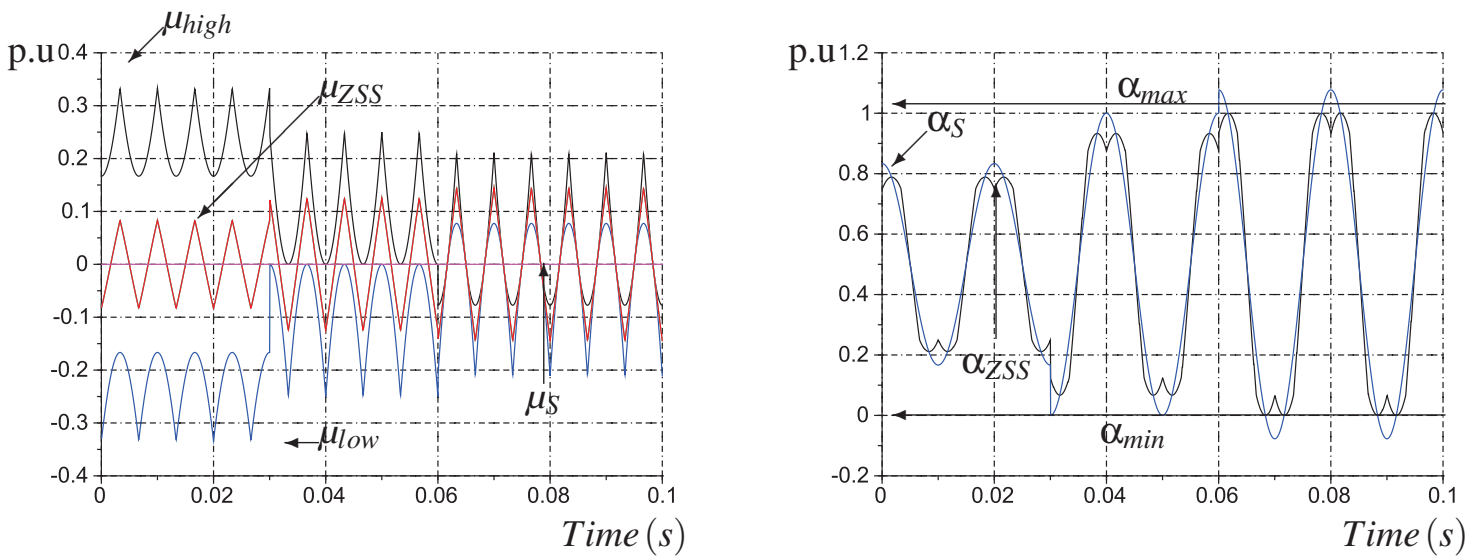

Figure 8: $\mu$ margins and $\mu$ waveforms expressed for Si- Figure 9: Duty cycle waveforms of the first VSI leg, nusoidal PWM - $\mu_{S}$ and Zero Sequence Signal PWM - expressed for Sinusoidal PWM - $\alpha_{S}$ and Zero Sequence $\mu_{Z S S}$.

Signal PWM - $\alpha_{Z S S}$.

\section{Switching losses}

For instance, while the linearity ratio is ensured, $\mu$ is now set in order to reduce the switching losses. Effectively, when the duty cycle $\alpha$ is set to 1 or 0 , the correponding VSI leg is not switched throughout a switching period $T_{s}$. Then the losses are naturally decreasing. Let us consider $\mu$ arbitrary fixed as

$$
\mu=\mu_{\text {high }}
$$

As illustrated in Fig. 10, the duty cylce waveforms change. For some switching period they are clamped to 1 . It is demonstrated in Fig. 11 that the general shape of the currents is still correct, whereas the leg
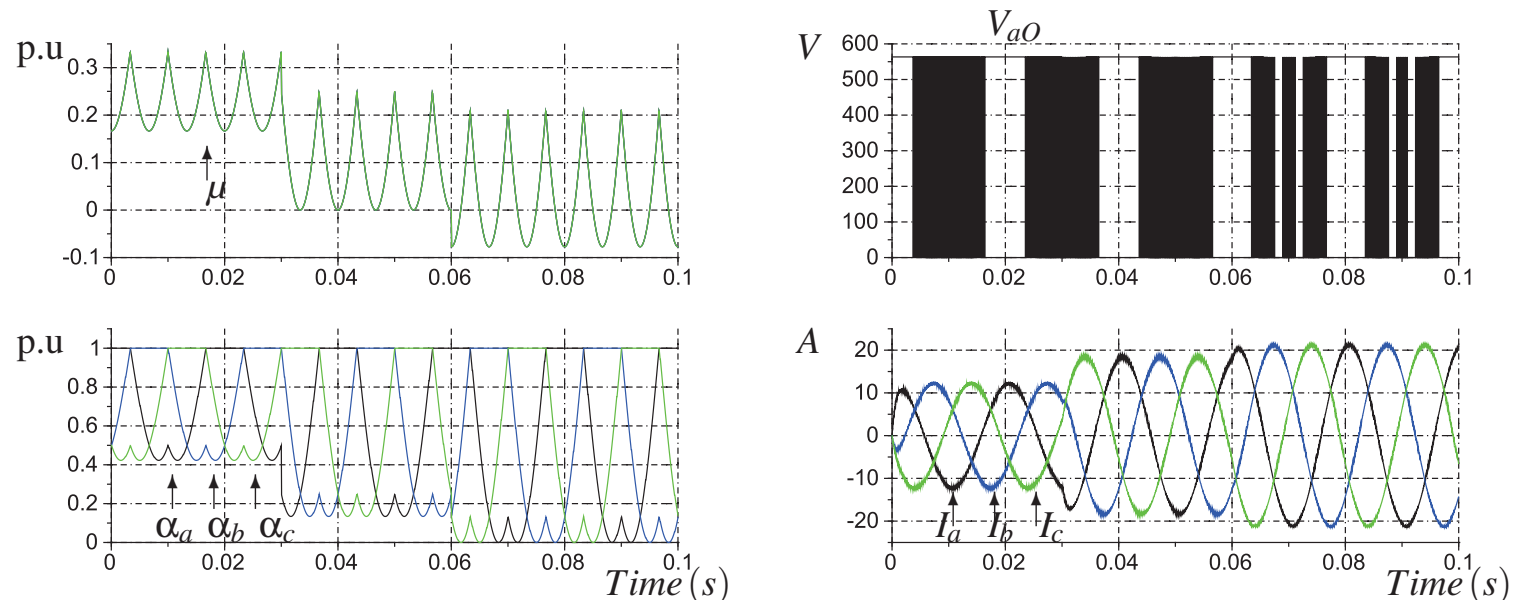

Figure 10: $\mu$ and duty cycle waveforms, taken for reduc- Figure 11: First leg voltage and three-phase currents obing switching losses.

tained with $\mu$ taken for reducing switching losses.

voltage is clamped to $E$ for more than one switching period. Of course it is linked with $\alpha_{i}=1$. In such example, if $\mu$ satisfies,

$$
\mu_{\text {low }} \leq \mu=\mu_{\text {high }},
$$

an optimized functionning is ensured as far as the linearity voltage zone and the switching losses are concerned.

\section{Conclusion}

In this paper a mathematical solution set for duty cycles used in carrier based PWM technique applied to Voltage Source Inverters is established. Using the generalized inverse theory, the solution set for the duty cycle is expressed. Two degrees of freedom that allows to describe some PWM published before are 
pointed out. Using matrix calculus and simulation software, it is demonstrated that each usual solution is included within admissible margins. This approach permits today to recover the known solutions but our works continue to explore new mathematical solutions that have interesting features as regards to the conversion of energy.

\section{References}

[1] Bimal K. Bose, Modern Power Electronics and AC drives, Prentice Hall PTR, 2002

[2] Ahmet M. Hava, and Russel J. Kerkman, and Thomas A. Lipo, Simple Analytical and Graphical Methods for Carrier-Based PWM-VSI Drives, IEEE Transactions on Power Electronics, vol. 14, january 1999, pp. 49-61

[3] Donald Grahame Holmes, The Significance of Zero Space Vector Placement for Carrier-Based PWM Schemes, IEEE TTransactions on Industy Applications, vol. 32 No 5, september 1996, pp. 1122-1129

[4] K. Zhou, and D. Wang, Relationship Between Space-Vector Modulation and Three-Phase Carrier-Based PWM: A Comprehensive Analysis, IEEE Transactions on Industrial Electronics, vol. 49february 2002, pp. 186-196

[5] S.R. Bowes and D. Holliday, Optimal Regular-Sampled PWM Inverter Control Techniques, Industrial Electronics, IEEE Transactions on", vol. 54, Issue 3, June 2007, pp.1547 - 1559

[6] M. P. Kaunierkowski, and M. A. Dzieniakowslu, Review of Current Regulation Techniques For Three-phase PWM Inverters, Proc. IEEE Industrial Electronics, Control and Instrumentation, 1994. IECON '94., 20th International Conference on, Bologna, Italy , september 1994, pp. 567-575

[7] Akshay K. Rathore and Joachim Holtz and Till Boller, Synchronous Optimal Pulsewidth Modulation for Low-Switching-Frequency Control of Medium-Voltage Multilevel Inverters, IEEE Transactions on Industrial Electronics, vol. 57 n 7, july 2010, pp. 2374-2381

[8] Poh Chiang Loh and D.G.Holmesand T. A. Lipo, Implementation and control of distributed PWM cascaded multilevel inverters with minimal harmonic distortion and common-mode voltage, Power Electronics, IEEE Transactions on, vol.20, Issue: 1 , january 2005, pp. 90 - 99

[9] Adi Ben-Israel and T. N. E. Greville, Generalized Inverses : Theory and Applications, Springer-Verlag, Second edition : 2003

[10] I. Fredholm, Sur une classe d'equations fonctionnelles, Acta Math., vol. 27, 1903, pp. 365-390

[11] E. H. Moore, On the reciprocal of the general matrix, Proc. Bull. Amer. Math. Soc. 26, 1920, pp. 394-395

[12] Adi Ben-Israel, The Moore of the Moore-Penrose inverse, Proc. Electron. Jour. Lin. Algebra. 9, 2002, pp. 150-157

[13] R. Penrose, A generalized inverse for matrices, Proc. Cambridge Philos. Soc. 51, 1955, pp. 406-413

[14] V. Lovass-Nagy, and R. J. Miller, and D. L. Powers, An introduction to the application of the simplest matrix-generalized inverse in systems science, Proc. IEEE Trans. Circuits and Systems, vol CAS-25, 1978, vol. 766-771

[15] Gene H. Golub and Charles F. Van Loan, Matrix computations, The John Hopkins University Press, Third edition : 1996

[16] Scilab: Le logiciel open source gratuit de calcul numerique, Scilab Enterprises, Orsay, France, 2012, http://www.scilab.org

[17] Marian P. Kazmierkowski, and R. Krishnan, and Frede Blaabjerg ”, Control in Power Electronics, Elsevier Inc., 2003

[18] Stephan Capitaneanu, and B. de Fornel, and M. Fadel, and J. Faucher, and A. Almedia, Graphical and algebrical synthesis for PWM methods, EPE Journal, august 2001, pp. 16-28 